\title{
7. The technological dimensions of governance
}

\section{TECHNOLOGICAL DRIVERS; TECHNOLOGICAL RESPONSES}

Again and again, technological innovations have driven the rise of new issue domains and, in the process, generated new needs for governance. Think of the machinery of industrial production, modern transportation systems, and advanced means of communication as sources of numerous examples. Prior to the machine age, the need to regulate industrial emissions of pollutants such as sulfur dioxide, nitrogen oxides, and greenhouse gases did not arise. The invention of trains, automobiles, and airplanes brought with it a host of governance questions pertaining to licensing, rules of the road, safety, and environmental impacts. Addressing needs for governance relating to telephones, radios, and television has produced a complex network of regulations dealing with matters like licensing and the allocation of broadcast frequencies along with the administrative apparatus needed to implement these regulations. There is nothing surprising or controversial about these observations. To be sure, efforts to develop and implement institutions to address these needs for governance have met with varying degrees of success. But there is no doubt about the existence of an intimate relationship between technological innovations and social institutions across a broad range of issue domains.

In this chapter, I take up several distinct aspects of the relationship between technology and governance. To begin with, there is the question of whether the processes involved in the development and application of new technologies are themselves governable. Is technological innovation a juggernaut that is unstoppable, without regard to the impacts of new technologies on human well-being (Smith and Marx 1994)? Or can we steer the course of technological innovation, encouraging the development of new technologies that seem likely to produce results that are beneficial in societal terms, while discouraging or even prohibiting work on technologies that seem likely to prove harmful to society? Conversely, there is the question of whether we can or should rely on the development of new technologies in our efforts to address various needs for governance. At the end of the day, can we count on techno- 
logical innovation to come to our aid as we endeavor to come to grips with complex problems like anthropogenic climate change? Or, as many believe, is the presumption that we can rely on what some commentators criticize as technological fixes to address such problems a dangerous habit that is likely to breed complacency in the face of novel needs for governance and to produce knock-on effects that generate new and sometimes more challenging needs for governance in the course of endeavoring to address existing needs (Commoner 1972)? More modestly, are there technological tools (e.g., advanced monitoring systems) that do not amount to technological fixes but that can play useful roles in conjunction with responses to needs for governance? Looming over all these concerns is the advent of the cyber age. Is the growing role of virtual reality, a phenomenon brought about by the development of a cascade of new information and communications technologies, a game changer generating novel needs for governance that existing institutions are not well positioned to address (Young, Yang, and Guttman 2020)? Will this result in growing challenges to the conventional assumption that we operate on a global scale within a political order in which membership is restricted to actors that are able to meet the requirements for recognition as sovereign states, and states are regarded as the principal actors when it comes to creating and implementing governance systems to address most needs for governance?

\section{CAN GOVERNANCE SYSTEMS STEER THE COURSE OF TECHNOLOGICAL INNOVATIONS?}

Much thinking about technological innovation reflects a sense of determinism (Heilbroner 1967). On this account, the processes involved in the development and application of new technologies exhibit a dynamic of their own that is largely beyond the control of governance treated as a social function involving efforts to steer human activities toward the production of outcomes that are desirable from a societal perspective. We regularly find ourselves seeking to address needs for governance in issue domains that arise as a result of the advent of new technologies. But the question I address in this section is whether the processes energizing the development of technological innovations are themselves ungovernable. Several distinct strands of thinking are embedded in this enquiry.

Whether their behavior is driven by the pursuit of material gain, the prospect of becoming famous, the desire to solve social problems, or just plain intellectual curiosity, individual inventors are motivated by a drive to figure out how things work and to explore opportunities to create new devices that can be put to practical uses. Thomas Edison, for example, turned technological innovation into a successful business, creating a large, well-staffed laboratory and coming up with numerous innovations that provided the basis 
for profit-making enterprises (Simmons 2016; Stross 2008). It seems likely, on the other hand, that the Wright brothers were seized with the challenge of building a machine capable of sustaining manned flight without regard to the commercial potential of their invention (McCullough 2016). The disconnect between innovation and eventual applications may be particularly pronounced in cases where scientific advances are critical to the achievement of success. Many of the atomic scientists, for instance, were drawn to participation in the Manhattan Project by the appeal of working on the cutting-edge scientific challenges involved in understanding nuclear fission in contrast to a practical desire to produce an atomic bomb. Leading scientists like Robert Oppenheimer became acutely aware of the moral and ethical issues associated with the development of nuclear weapons, largely in retrospect once the genie was out of the bottle and the nuclear age was under way. Similar observations may apply to the work of some scientists engaged in the production of knowledge underlying the development of the capacity to engage in heritable human genome editing today (Doudna and Sternberg 2017; Isaacson 2021). In all these cases, the implication is the same: it is difficult, if not impossible, to steer the behavior of those whose efforts give rise to technological innovations.

Beyond this, the imperatives of competition often drive the development of technological innovations. Companies invest substantial resources in research and development (R\&D) to generate ideas for new products that will give them a competitive edge in their interactions with rivals. States engage in arms races in order to prevent opponents from gaining the upper hand due to technological innovations and subsidize industries to help them produce technological breakthroughs expected to promote the national interest as well as corporate interests. As the example of arms races makes clear, there are instances in which the resultant competition erodes the security of all the participants and destabilizes the larger system of which they are a part. But that does not alter the fact that what some have characterized as the "mad momentum" arising from competition can become a powerful driver of technological innovation in a variety of settings. The result is a sense that while we may be able to address needs for governance generated by the advent of new technologies, we have little capacity to steer the processes that produce technological innovations.

Nevertheless, it is easy to exaggerate the force of these arguments regarding technological determinism. To begin with, many of the devices inventors create are of little or no interest to others or fail for one reason or another to gain traction within society at large. In Los Angeles, there is a Museum of Jurassic Technology that displays a wide variety of innovative devices that have fallen by the wayside for the most part, never to be heard from again. The point is that a large proportion of the results of technological innovation turn out to be more or less irrelevant from the perspective of governance. What is more, while inventors may be more imaginative than others, they are not 
immune to the influence of material incentives or to broader social and cultural currents. Much of the work of inventors is made possible by the provision of material resources on the part of companies or government agencies interested in the development of technologies designed to solve specific problems or to achieve particular goals. While it may not be easy to govern the actions of those in a position to make decisions about the allocation of such resources, there is certainly no basis for arriving at a general conclusion that decisions of this sort are ungovernable. No doubt, the effectiveness of governance in this realm is a variable affected by a variety of factors. But this does not support any general conclusion about technological determinism.

An interesting issue in this realm relates to deliberate efforts to prohibit or limit the development of new technologies as a matter of public policy. The horrors associated with the use of poison gas as a weapon on the battlefields of World War I, for example, stimulated a concentrated effort to ban work on technological innovations that could lead to the development of various types of chemical and biological weapons. Although the resultant 1925 Geneva Protocol banning the use of poison gas has not been completely effective, there is little doubt that this initiative and several others that followed have made a significant difference. More recently, there has been a focused effort to place sharp restrictions on efforts to develop effective anti-ballistic missile systems that many regard as having the potential to disrupt the strategic balance among the nuclear powers, giving rise to extremely unstable and dangerous developments in great-power politics. It would be a mistake to conclude that these efforts to control technological innovation regarding weapons systems have been entirely effective or to assume that ongoing efforts of this kind will prove highly effective in the future. As in other domains, efforts to create and implement governance systems to steer the trajectory of technological innovation often run into problems; they may fail altogether in some instances. But this is not the same as arguing in more general terms that there is something in the nature of technological innovation that makes it ungovernable.

A more fruitful topic for consideration may be experience gained from the development of mechanisms designed to steer the activities of those engaged in processes of technological innovation. To begin with, it is apparent that governments play prominent roles in steering the trajectory of technological innovations in a variety of issue domains. There is a long history of such interventions. A famous case features the British Parliament's action in 1714 to establish a prize of $£ 20,000$ to be awarded to anyone able to create a device capable of measuring longitude at sea accurate within half a degree, an initiative that led eventually to the development of the chronometer (Sobel 1995). Not surprisingly, many of the initiatives of governments are driven by concerns about national security. Governments regularly allocate large sums to support R\&D intended to produce more advanced weapons systems, 
such as sophisticated aircraft, advanced ballistic missiles, and more powerful communications systems. Whatever their usefulness with regard to military security, the resultant technologies produce applications that have far-reaching consequences for civil society. Many of the technologies embedded in sophisticated civilian aircraft today originated in efforts to develop advanced military aircraft. The rockets we use to launch civilian payloads into space owe their existence to the development of technologies for military purposes. A particularly prominent illustration involves the origin of the internet, which grew out of work conducted under the auspices of the US Department of Defense's Advanced Research Projects Agency (DARPA). Of course, this development has triggered an extraordinary flow of additional innovations that go far beyond the intent of the original DARPA initiative (DeNardis 2014). But it remains the case that all these developments stem from a conscious effort to steer the course of technological innovation.

Nor are such efforts limited to interventions inspired by the pursuit of national security. Governments regularly act to promote technological innovation in a variety of areas, using policy instruments ranging from tax breaks to measures designed to protect companies from foreign competitors to outright subsidies. An interesting contemporary example involves the provision of incentives for those engaging in R\&D in the realm of technologies relevant to renewable energy. These incentives range from support for producers interested in pursuing technological advances in the fields of solar and wind power to support for consumers (e.g., rebates on electric cars, financial incentives for installing solar collectors) that translates into support for those engaged in the development of new technologies. Of course, it is important to recognize that these forms of support for technological innovation are sometimes offset by a variety of subsidies that support continued reliance on fossil fuels in the energy-intensive sectors of modern societies. Nevertheless, this does not change the observation that the actions of governments play a significant role in determining the trajectory of technological innovation in many issue domains.

Beyond this, governments develop and administer a wide range of regulations that have far-reaching impacts on the trajectory of technological innovation on the part of private or nongovernmental actors working in many areas. While they differ from place to place, almost all societies have developed systems of patents, licensing arrangements, health and environmental standards, and liability rules designed to steer processes of technological innovation. The availability of patents, for example, encourages inventors by offering them rights that can and often do result in streams of income accruing to those who hold them. There is great variation among societies in the character of their systems of patent law. Some influential individuals even argue that inventions should be shared freely with all members of society (Hyde 2010). 
But, for the most part, recent trends in patent law have tended to strengthen the rights of those who hold patents. Licensing, by contrast, may operate as a restraint on the activities of inventors. In cases where inventors are required to obtain licenses in order to market their innovative technologies, for instance, society is able to impose a variety of restrictions relating to health and safety, employment, and more general ethical concerns.

Because new technologies often generate unintended side effects, governance systems frequently include regulations intended to suppress negative externalities or to ensure that developers and users of technological innovations have incentives to minimize their unwanted side effects. Much the same is true of liability rules that require users of new technologies to compensate those harmed by the development and application of innovative technologies. The stringency of such rules varies greatly across societies and even across issue domains within the same society. What is more, numerous analysts have shown that the difficulty of pinning down causal connections frequently makes it difficult for victims to obtain adequate redress for harms generated at least in part by the introduction of new technologies (Fagin 2015). Nevertheless, it is fair to conclude that societies use a variety of institutional arrangements to guide the behavior of inventors and that these arrangements do make a difference under a variety of circumstances, even though their effectiveness in any given setting is far from assured.

A particularly challenging situation with regard to governance arises in cases involving what are commonly referred to as dual-use technologies. These are technologies that have multiple uses, some of which are deemed socially beneficial while others are widely regarded as harmful. Perhaps the classic example is nuclear energy, where peaceful uses are generally treated as desirable but military uses in such forms as the development of weapons of mass destruction are seen as harmful. But there are other prominent cases, including biotechnologies that can enhance public health but also have the potential to be used for harmful purposes by antisocial actors, and information technologies that allow actors to engage in all sorts of productive activities but that also open up possibilities for cyberterrorism and cyberwarfare. From the perspective of governance, the challenge is to find ways to permit or even encourage socially beneficial uses of such technologies, while at the same time suppressing or sharply limiting harmful uses. The track record resulting from efforts to solve this puzzle is not encouraging. The rapid growth of misuses of cyber age technologies ranging from identity theft to illegal interventions in electoral processes and various forms of cyberwarfare is a daunting case in point (Perlroth 2020). Today, we are facing a variety of severe challenges relating to the regulation of uses of biotechnology in the absence of any well-conceived governance strategies likely to prove effective (Evans 2020). But this is no reason to throw up our hands in the face of the forces of techno- 
logical determinism. As I argued in Chapter 2, these developments are among the grand challenges of global governance in the 21st century; efforts to devise new strategies for steering behavior in these areas will constitute cutting-edge concerns for those who work on the creation and implementation of governance systems for some time to come.

The argument of this section supports the conclusion that governance systems are often significant and sometimes decisive determinants of the trajectory of technological innovation. But it also suggests three broader observations that are important in providing context for this conclusion. As is the case in all other domains, the impact of governance systems is only one of a number of factors that together determine the trajectory of technological innovations. There is ample scope for asking questions about what works and does not work in this realm and thinking about opportunities for enhancing the effectiveness of governance systems in specific settings. It is worth noting that there are significant normative issues in this realm. For example, there are those who argue that technological dynamism is generally good for society and that we should not encumber inventors with restrictions or limitations likely to hinder the course of technological innovation. Without going into detail here, it seems reasonable to suggest that the trick is to strike a balance between the imposition of appropriate restrictions (e.g., limitations designed to protect public health) and the avoidance of restrictions (e.g., oppressive licensing requirements) likely to stifle the development of socially beneficial inventions. But applying this general standard to specific situations will give rise to intense controversy in many cases.

A second observation is that effective efforts to steer the course of technological innovation can lead to outcomes that many will find objectionable on ethical or moral grounds. Perhaps the most prominent cases center on the role of the state in allocating public revenues to support technological innovations justified in the name of national security. Major powers provide massive support for R\&D relating to the invention of ever more sophisticated weapons systems that are said to be needed to defend society against the actions of hostile outsiders. But in most cases, the effect is to stimulate arms races that leave no one more secure and everyone with fewer resources to invest in the promotion of human well-being. But national security is not the only realm in which this concern arises. In modern times, for example, societies have supported the development of a variety of technologies that are major contributors to the onset of anthropogenic climate change, thereby accelerating the emergence of one of the grand challenges of global governance in the $21 \mathrm{st}$ century. Of course, there is room for disagreement about such matters. What some see as negative results of the efforts of governments to steer the course of technological innovation, others will treat as socially desirable. But for now, the point is that there is a critical difference between effectiveness and 
social desirability when it comes to evaluating the results of efforts to steer the trajectory of technological innovation.

Finally, it is useful to draw a distinction between the results of efforts to steer the trajectory of technological innovation in specific instances and the broader forces of technological dynamism. It may be that while governance works in specific instances (e.g., prohibiting the development of certain types of biological weapons), the forces of technological determinism are difficult to control on an aggregate or systemic level. This is the concern of those who argue that at the macro level the technological juggernaut is uncontrollable and that the best we can hope for is to find effective ways to adjust to a continuing wave of technological innovations. This is an important line of thinking (Winner 2020). But it is difficult to analyze such arguments on a systematic basis. It is probably fair to say that some societies (e.g., America in the 20th century) are more in thrall to the forces of technological determinism than others. And it is certainly true that there are waves of technological innovation in the sense that the pace of innovation varies greatly across issue domains, social contexts, and time periods. But none of this reduces the importance of asking questions about the effectiveness of various strategies for governing the course of technological innovation in specific times and places.

\section{CAN TECHNOLOGICAL INNOVATIONS HELP IN MEETING NEEDS FOR GOVERNANCE?}

A wide chasm separates the views of those who believe technological innovations can produce solutions to problems giving rise to needs for governance from the views of those who regard technological innovations as sources of problems and technological solutions as unsatisfactory at best and likely to generate serious knock-on problems under the most favorable circumstances. On one side are those who regard necessity as the mother of invention. They argue that acute needs for governance will incentivize inventors and that human ingenuity is sufficient to come up with solutions to most - possibly all - problems when the incentives are right. Their opponents are likely to deride such thinking as constituting a form of technological salvationism apt to eventuate in ill-considered technological fixes that offer no more than partial solutions to current problems and that are unlikely to contribute to human well-being in the long run. Stated in their strongest terms, these opposing perspectives often stimulate acrimonious debates among proponents that fail to contribute to a more nuanced assessment of the contributions of technological innovations to meeting needs for governance in modern societies.

It is not difficult to find concrete examples that illustrate the arguments of the two camps. A well-known example centers on the looming crisis of increased horse manure in urban streets toward the end of the 19th century. The introduc- 
tion and rapid growth in the use of motorized vehicles led to the evaporation of this crisis over the span of a few years. But counter examples are equally easy to find. The development of tall smokestacks as a response to the effects of local air pollution generated by power plants and factories, for instance, produced the phenomenon of long-range air pollution, introducing the problem of acid precipitation and eventually leading to the negotiation of international agreements intended to curb the impacts of long-range transboundary air pollution. Problems with chemicals (e.g., ammonia) used in refrigeration provided the impetus for the development of chlorofluorocarbons (CFCs) during the 1930s. But the new chemicals turned out to be ozone-depleting substances that caused a serious thinning of the Earth's stratospheric ozone layer. The result was an acute need for governance leading in due course to the negotiation of the 1987 Montreal Protocol on Substances that Deplete the Ozone Layer followed by a series of substantive amendments strengthening the provisions of this agreement in the following years (Parson 2003). An interesting feature of this example is that the success of the Montreal Protocol, widely regarded as one of the most effective international regimes of modern times, is linked closely to the ability of major corporate players like DuPont to come up with alternative technologies when confronted with powerful incentives to phase out the production and consumption of ozone-depleting substances, including CFCs, halons, and several other families of chemicals.

What these observations suggest is a need to think systematically about the relationship between technological innovation and the creation and implementation of social institutions, including a consideration of the conditions that determine whether technological innovation becomes part of the problem, part of the solution, or both in situations featuring needs for governance. Setting aside the extreme views - technological innovations can solve all problems versus "tech fixes" are part of the problem - questions about the interactions between technological innovations and the creation and administration of governance systems come into focus. Can technological innovations contribute to the development of steering mechanisms that make it possible to alleviate or even solve problems giving rise to needs for governance? Can governance systems channel efforts to develop technological innovations, promote the development of some innovations in contrast to others, or impose regulations channeling the application of innovations to specific issues? In short, the interesting questions have to do with the interactions between technology and social institutions rather than whether we should treat the promotion of technological innovations or the creation of new institutions as alternative ways to respond to needs for governance. One way to explore this theme systematically is to examine conditions that are likely to determine whether technological innovations constitute a help or a hindrance when it comes to addressing various needs for governance. 
One important condition involves what many analysts call moral hazard (Arrow 1971). The issue here is easy to identify but difficult to evaluate with reference to specific situations. If actors ranging from private individuals to prominent political leaders believe that technological innovations will come to the rescue once a problem becomes sufficiently severe, will this generate complacency regarding the creation and implementation of governance systems designed to steer behavior toward the avoidance of largescale problems or toward the development of solutions that do not rely on technological fixes? In the case of climate change, for example, does the idea that we can resort to various forms of geoengineering undermine or erode incentives to strengthen the provisions of the 2015 Paris Climate Agreement? Or can we move vigorously to build an effective climate regime, holding in reserve the option of turning to various forms of geoengineering if and when we reach a point where the case for adopting technologies of this sort becomes compelling? There are no simple answers to these questions. No doubt, complacency is a serious concern. It will affect the thinking of some actors more than others. A key question in this regard concerns the prospect of taking steps to explore the pros and cons of technological options (e.g., various forms of solar radiation management), while at the same time making it clear this is not an alternative to proceeding energetically to improve institutional arrangements like the Paris Climate Agreement. At the end of the day, this is a challenge of creating an appropriate mindset regarding the contributions of technological innovations rather than a technical challenge (e.g., creating effective vaccines for Covid-19) to which there is a well-defined solution. But that does not mean we cannot make progress toward meeting this challenge.

Another condition regarding the role of technological innovations has to do with the occurrence of harmful side effects arising from the development and application of technologies designed to deal with societal problems. All major technological innovations produce both direct and indirect effects. The development of solar panels to increase the use of renewable energy or electric vehicles to reduce the use of gasoline, for example, has triggered the creation of new companies, introduced new types of jobs, and changed the behavior of investors regarding the allocation of available capital. A variety of indirect effects are likely to follow, including the loss of jobs for workers in old industries and the decline of communities that are unable to adjust easily to the shifting landscape of industrial activities (e.g., cities in the American Rust Belt). Some of these consequences may prove costly to those whose livelihoods are tied to old technologies superseded by the development and application of technological innovations. There is nothing surprising about occurrences of this sort; they are classic consequences of technological innovation at all times and in all places. Societies may want to assist those displaced by such changes to improve their chances of finding new roles or even compensate victims. 
But there is no reason to adopt measures to block or slow down the course of technological innovation for this reason alone.

The concern about relying on technological solutions to address problems producing needs for governance centers on two more specific issues. One of these issues concerns unforeseen harms or costs that are not taken into account in decisions regarding the introduction of technological innovations in efforts to meet needs for governance. For example, no one foresaw or fully understood the array of environmental impacts associated with the operation of nuclear power plants or the costs of decommissioning such plants at the end of their productive lives in early discussions regarding the role that nuclear energy could play as an alternative to systems relying on fossil fuels to generate electricity. We have been slow to recognize, much less to address adequately, the harms that increased reliance on hydroelectric power impose on those displaced as a result of the construction of large dams and on the surrounding ecosystems. These are specific externalities resulting from efforts to reduce emissions of greenhouse gases rather than normal effects associated with technological innovations under any circumstances. They deserve to be taken seriously in any assessment of the pros and cons of adopting technological responses to societal problems.

Then there are issues regarding the extent to which technological responses constitute full-fledged solutions to the problems at hand. Consider again the case of geoengineering as a response to climate change (National Research Council 2015). Making use of technological innovations to manage solar radiation might suffice to control the increase of temperatures at the Earth's surface by blocking some incoming solar radiation. But so long as such measures are not accompanied by a substantial reduction in the use of fossil fuels, they would provide no solution to the disruptions caused by the acidification of marine and terrestrial ecosystems. What is more, relying on solar radiation management to address the problem of climate change would require what amounts to a permanent commitment. Any interruption in the application of the relevant technologies would be followed by a rapid rise in temperatures at the Earth's surface with consequences that might well be catastrophic. While turning to geoengineering as an emergency response to a problem that we have allowed to get out of control may seem unavoidable, therefore, no one should be under the illusion that this technological fix constitutes a fully satisfactory response to the problem of climate change. Certainly, it offers no basis for relaxing our efforts to introduce institutional arrangements that will lead to reductions in emissions of greenhouse gases.

Sometimes technological solutions generate new and potentially more serious problems as an unintended and typically unforeseen consequence of efforts to come to terms with the initial problem. I have referred already to the examples of acid rain and the thinning of the stratospheric ozone layer. 
But it turns out that these cases are not unusual. Other examples are easy to identify. The development and application of chemicals such as DDT have proven highly effective in combatting diseases like malaria. But these persistent organic pollutants are highly destructive in ecological terms (Carson 1962). They have contributed to the profound problem we now understand as the loss of biological diversity, and efforts to control uses of this large family of chemicals have given rise to international agreements like the Stockholm Convention on Persistent Organic Pollutants. Similar remarks are in order regarding what is known as the Green Revolution, a development that undoubtedly has played a role in reducing hunger on a global scale but that has also contributed to the loss of biological diversity due to increased reliance on a small number of cultivars and to growing reliance on chemical fertilizers and pesticides (Mann 2019). The fact that the application of technological innovations to meet needs for governance can and often does generate knock-on problems does not constitute a reason for avoiding all technological responses to the world's problems. But it does provide a compelling reason to proceed with an abundance of caution in turning to technological innovations to meet a wide range of needs for governance.

In the final decades of the 20th century, the idea of technology assessment became popular as an approach to thinking systematically about the benefits and costs of turning to technological responses in making informed decisions about matters of public interest, including responses to needs for governance. In 1972, for example, the US Congress created an Office of Technology Assessment to "provide Congressional members and committees with objective and authoritative analysis of the complex scientific and technical issues of the late 20th century." The assumption embedded in such measures is that we can make intentional choices regarding the role of technology in dealing with matters of public policy and that it is possible to represent all the consequences associated with available options in terms of a utilitarian and preferably quantitative calculus of benefits and costs. This should make it a relatively easy matter to select that option in any given situation with the highest benefit/cost ratio. Whatever its appeal in conceptual terms, the effort to apply technology assessment to issues regarding the role of technological innovations in meeting needs for governance has produced disappointing results. In many cases, the consequences of technological solutions to societal problems include major unknowns and even unknown unknowns. The idea that it is feasible to represent the consequences of technological solutions in terms of some common metric of benefits and costs has proven deeply controversial at best. What is more, the application of benefit/cost analysis has failed to suppress continuing efforts on the part of interest groups to promote the selection of options favorable to themselves, regardless of the consequences for society as a whole. As a result, what began as a promising new approach to policy analysis has 
fallen by the wayside. In the United States, Congress dismantled the Office of Technology Assessment in 1995, following the 1994 election, which brought to power Republican legislators who regarded the office "as wasteful and hostile to GOP interests." With regard to the specific issue of evaluating the contributions of technological innovations to meeting needs for governance, it is clear that we cannot count on technology assessment as a rigorous procedure for examining the conditions under which technological solutions are feasible, much less desirable.

Much of the debate about technological solutions has turned on questions of effectiveness. Can geoengineering solve the climate problem at an affordable cost? Would relying on geoengineering to solve the climate problem produce knock-on problems, so that the cure might turn out to be at least as bad as the ailment over time? But it is increasingly clear that there are important normative issues associated with a turn to technological innovations to meet needs for governance. In some cases, these issues feature matters of justice or fairness. Do technological solutions in specific cases allow the rich and powerful to protect themselves from the negative impacts of environmental problems, while leaving the masses to suffer the consequences? Are technological solutions that offer protection to those living today likely to impose severe costs on members of future generations? At the same time, there are larger normative questions that come into focus in thinking about the role of technological innovations in meeting needs for governance. Does the idea that we can count on technology to come to our rescue in addressing issues like anthropogenic climate change reflect a kind of technological salvationism that encourages us to avoid the hard work of thinking carefully about the consequences of relying on energy produced through the combustion of fossil fuels? More broadly, does technological salvationism breed an attitude of indifference or complacency regarding the disruptive consequences of human activities that seem appealing in terms of personal gratification?

If these concerns are taken seriously with regard to the contributions of technological innovations to solving specific problems, the issues are magnified greatly when technological salvationism becomes a pervasive feature of the prevailing culture in major societies like the United States or China or in international society more generally. What is at stake here is a clash between the values and practices of technological cultures, which set store by a continuous flow of technological innovations to provide new experiences and solve old problems, and more humanistic cultures, which emphasize the distinction between humans and machines and seek to promote practices (e.g., interest in the arts, mindfulness) that do not rely on technological innovations to generate new experiences. Of course, it is easy to exaggerate this cultural divide, and there is no way to reach conclusions regarding the relative merits of one cultural orientation or the other in any case. Nevertheless, it is worth noting 
that any comprehensive assessment of the role of technological innovations in meeting needs for governance must take into account the links between this specific concern and a range of issues regarding the links between technology and culture.

This broader concern will resurface in the concluding section of this chapter on consequences arising from the onset of the cyber age. But one observation that seems relevant at this stage is that there may be a growing need for governance regarding efforts to make use of technology in meeting needs for governance (Royal Society 2009). Once again, the case of geoengineering offers a clear example. Who should decide when to resort to geoengineering to alleviate the impacts of anthropogenic climate change and whether some forms of geoengineering are more acceptable than others? Should we devise different rules to deal with those measures commonly referred to as carbon dioxide removal in contrast to solar radiation management? Should we allow research regarding various types of solar radiation management to go forward with few restrictions, but then shift gears when it comes to the deployment of the relevant technologies on a sizable scale? Would it be helpful to articulate the provisions of a regime for geoengineering in the form of an international legally binding instrument? How might we address the challenges of moving such a regime from paper to practice? While geoengineering constitutes a prominent case of great interest to analysts and practitioners today given our inability to come to grips with the problem of reducing emissions of greenhouse gases sufficiently to address the problem of climate change, it is by no means unique. It is not difficult to think of other examples relating to the $21 \mathrm{st}$ century's grand challenges of planetary governance, such as the possibility of making use of technological innovations in the effort to come to terms with the dramatic growth in disruptive misuses of cyberspace.

\section{CAN TECHNOLOGICAL INNOVATIONS IMPROVE THE PERFORMANCE OF GOVERNANCE SYSTEMS?}

Setting aside for the moment the debate about the virtues of technological solutions and the dangers of technological fixes, we can turn to a series of more concrete issues regarding roles that technology can play in the development and administration of institutional arrangements dealing with a variety of needs for governance. Here, it is helpful to make use of the idea of the policy cycle as a means of organizing the discussion. Technology may prove useful across the various stages in the cycle, ranging from the identification of emerging needs for governance through the creation of governance systems and their movement from paper to practice and on to the evaluation of performance 
and the consideration of adjustments to existing systems needed to improve performance in subsequent time periods.

Advanced observing systems can provide early warning regarding the emergence of needs for governance and the assessment of competing arguments regarding the sources and seriousness of the relevant problems. Airborne and satellite-based observations have played key roles in the identification of the problems of acid rain, the thinning of the stratospheric ozone layer, and climate change. Similar observations are in order regarding the pollution of rivers and lakes, the destruction of coral reefs as a consequence of the acidification of sea water, and the spread of radioactive fallout from accidents involving nuclear power plants at Chernobyl, Three Mile Island, and Fukushima. Growing awareness of the problem of plastic debris today is made possible through the application of technological innovations allowing us to track the debris over long distances and in remote places. There is every reason to expect that technological innovations will become more and more prominent, both in detecting and in analyzing the nature of emerging needs for governance during the foreseeable future.

Technology can also provide assistance in designing the provisions of governance systems to address what we commonly refer to as the problem of fit (Young 2002). Computer programs can provide assistance in making decisions regarding the location of the boundaries of marine protected areas in such a way to achieve common goals while minimizing harm to the interests of individual stakeholders. Integrated assessment models can help to set targets for reductions in emissions of greenhouse gases by making projections regarding the relationship between emissions and the concentration of greenhouse gases in the Earth's atmosphere. Remote sensing can help to identify what areas need to be included within the scope of systems designed to protect marine mammals from harmful ship strikes. None of these applications of technology eliminates the need for policymakers to make hard choices. But they can contribute to the pursuit of informed decision-making on the part of those charged with the management of complex systems.

Likewise, technology can play a number of important roles when it comes to moving the provisions of governance systems from paper to practice. Some of these roles center on issues of monitoring, reporting, and verification. In cases where onsite monitoring would be difficult due to remoteness in spatial terms or sensitivity in political terms, satellite-based monitoring systems often are able to generate the information needed to facilitate the administration of governance systems. Automatic information systems can determine whether ships at sea are located in areas subject to various regulatory measures. Remote sensing can help to determine trends in phosphorus levels in major water bodies. Many assessments of the performance of international governance systems have noted deficiencies in the actions of members when it comes to 
fulfilling reporting requirements. This can become a serious problem when it comes to the administration of regimes. But technological innovations involving the growing sophistication of Earth observing systems can help to alleviate this problem, without precipitating political battles or triggering sensitivities arising from efforts to bring pressure to bear on regime members to fulfill reporting requirements.

As this discussion suggests, technological innovations may prove helpful as well in addressing problems of compliance, even in a politically decentralized setting like international society. Consider the case of the Polar Code dealing with commercial shipping in Arctic waters, which entered into force at the beginning of 2017 (Hildebrand et al. 2018). The code requires ships sailing in Arctic waters to be in possession of a valid license to operate in the form of a Polar Certificate and to have an operational transponder on board at all times. Satellite-based automatic information systems can track the movements of specific ships in Arctic waters in real time. Monitors can then match the resultant observations with an electronic database to determine whether individual ships are in possession of a valid license. Those who manage the data can share this information with port authorities, so that these authorities will know whether a ship is in compliance with the rules and can act accordingly when the ship's captain or owners request permission to enter a specific port. This example is specific to a particular case. But it illustrates the proposition that technological innovations can play a role in helping to ensure compliance with the provisions of governance systems, even in settings like international society where there is no government in the ordinary sense of the term to deal with matters of compliance and enforcement.

Technology may also come into play in assessing the performance of governance systems to determine whether there is a need to strengthen the provisions of such systems to meet the relevant needs for governance. The 2015 Paris Climate Agreement, to take a prominent case, calls for actions on the part of members to limit temperature increases at the Earth's surface to $2^{\circ} \mathrm{C}$, and if possible to $1.5^{\circ} \mathrm{C}$. To this end, the parties to the agreement have pledged to make what are known as Nationally Determined Contributions and to review these contributions at regular intervals to determine whether they need to be strengthened. In this connection, it is easy to see that technologically sophisticated observing systems will be essential in tracking performance, providing the information needed to drive what the agreement calls the Global Stocktake process. While there may be debates about the relationship between rising concentrations of greenhouse gases in the Earth's atmosphere and temperature increases at the surface of the Earth, there can be no doubt about the critical role that sophisticated technology can play in evaluating the performance of this governance system over time. 
What emerges from this discussion is a close connection between governance and technology across the full range of phases of the policy cycle from the identification of new needs for governance to the evaluation of the performance of governance systems that have been in place for some time. Importantly, nothing in this discussion raises questions about whether we are in danger of becoming dependent on technological fixes that turn out to do more harm than good in the long run. But it does suffice to demonstrate that there are intimate connections between social institutions and technologies, especially when it comes to crafting governance systems that are well matched to the character of the problems at hand and assessing the performance of the resultant systems once they are up and running.

\section{IS THE ONSET OF THE CYBER AGE A GAME CHANGER?}

A cascade of technological innovations over the course of the last four to five decades has triggered the onset of the cyber age (Isaacson 2014; Young, Yang, and Guttman 2020). Including developments in software as well as hardware, this cascade has already produced computers, smart phones, the internet, the world wide web, social media, robots, artificial intelligence, machine learning, big data, the internet of everything, and facial recognition. And there is no indication that the flow of new innovations will slow during the foreseeable future. What sets this cascade of developments apart from other technological innovations is that the new technologies highlight the role of virtual reality in contrast to material (biophysical and socioeconomic) reality. Increasingly, human activities feature virtual interactions in the sense that people are able to engage in a growing range of activities making use of digital technologies without engaging in any face-to-face interactions. Many are able to work from home, interact with one another for business or pleasure using virtual platforms, and handle a large proportion of their needs via online transactions. The eruption of the Covid-19 pandemic in 2020 clearly has accelerated and intensified this trend. But there is no doubt that the trend toward reliance on digital technologies using virtual reality to deal with a growing range of tasks will continue even after the pandemic subsides.

Two additional features intensify the distinctiveness of the cyber age. One centers on the pace of innovations in the realm of digital technologies. The flood of technological innovations has reached a point where even the most technologically savvy individuals find it difficult to keep up with new developments arising in a variety of fields. We are all in danger of falling further behind in relation to the cutting edge of technological innovations. In addition, private individuals working in remote locations with relatively few resources are able to generate innovations that mushroom into digital technologies 
becoming important features of the cyber age. Think of the origins of Google and Facebook as examples of this phenomenon. Among other things, this feature of the cyber age makes it difficult for public authorities to control the pace and trajectory of technological innovations, no matter how determined they are to do so. Adding these features together suggests that we are now operating in a setting that differs qualitatively from the setting existing prior to the onset of the cyber age. What are the implications of this shift for the generation of needs for governance and the creation of governance systems to meet these needs?

For one thing, there is the issue of developing governance systems to manage human interactions involving the use of the internet, the world wide web, social media, and so forth. As others have shown, early efforts to deal with this issue involved the evolution of social practices through processes that were to a large extent spontaneous. The development of a suite of practices required for the operation of the internet on an orderly basis provides a striking example (Brousseau et al. 2012; DeNardis 2014). But with the passage of time, we are witnessing a rapid growth in misuses of cyberspace to pursue disruptive and largely illegitimate ends. These misuses range from the activities of individuals involving identity theft, bullying, the tarnishing of personal reputations, and the willful disruption of business operations to the activities of organized groups featuring corrupt financial practices, interventions in electoral processes, and cyberterrorism and on to the activities of government agencies bent on weakening opponents and engaging in what is generally regarded as cyberwarfare. The net result is that what began as a remarkable example of the rise of spontaneous order has devolved into a situation featuring a growing range of needs for government that are almost certainly impossible to fulfill spontaneously and that pose fundamental challenges for those seeking to develop intentional governance systems. At this juncture, most of us are still able to use the internet and related digital systems without incident on a day-to-day basis. But the emerging challenges in this realm are daunting (Perlroth 2020).

What are the prospects for governing or steering the ongoing flow of innovations in the realm of digital technologies? Given the variety of new developments and the pace at which they arise together with the facts that innovations are often the work of small groups of individuals who work in isolated locations and are able to launch their innovations on virtual platforms, the prospects for efforts to control the continuing cascade of innovations in the realm of digital technology seem dim. Consider the history of developments like YouTube, Twitter, Instagram, and TikTok in this connection. It is true that major players like Alphabet, Facebook, Microsoft, and Alibaba typically buy up innovations once they show clear signs of success, giving these large corporate players some ability to guide the trajectory of developments in the 
realm of digital technology. Nevertheless, the major players do not control the initial stage of the innovative process, and in any case there is no reason to assume that these corporate players are motivated by a desire to steer developments toward socially desirable outcomes. Recent controversies regarding the extent to which platforms like Facebook and Twitter should be required to assume some responsibility for the content of material appearing on their platforms (e.g., hate speech, falsehoods intended to sway voting behavior) are particularly interesting in this connection.

On the other hand, public authorities may be able to exercise control over applications of digital technologies, even if they have difficulty controlling innovative processes themselves. Perhaps the most far-reaching example in this regard centers on China's efforts to construct a Great Firewall to limit the access of its citizens to digitally distributed information and to make use of digital technologies allowing for the use of big data and facial recognition software as instruments of social control. China has invested heavily in these efforts, and there is no doubt that these efforts have produced significant results (Klimburg 2017). For example, China blocks the access of ordinary citizens to products distributed by Google, takes down materials considered undesirable (e.g., the report on climate change known as Under the Dome) shortly after they are uploaded, and engages in surveillance of the activities of individual citizens.

Nevertheless, it is worth noting how difficult it is to achieve success in such efforts to control applications of digital technologies. Anyone in China who is willing to invest time and energy can overcome the restrictions of the Great Firewall. The installation of a VPN allows access to restricted materials. Users of WeChat, the Chinese equivalent of WhatsApp, can communicate freely with people all over the world. There is no problem including Chinese participants in virtual meetings using platforms like Zoom and Microsoft Teams. Even internally, there is a cat-and-mouse game in which clever users of digital communications systems engage in the invention of new terminology to replace terms that have been censored by the authorities. Clearly, this extraordinary campaign to control the application of digital technologies has achieved results, especially when it comes to the day-to-day interactions of ordinary people. Still, it is remarkable how easy it is for those who are motivated to overcome or circumvent these restrictions on a regular basis.

So, what about the role of digital technologies in solving societal problems or helping to meet needs for governance in various issue domains. I have commented already on the difficulties involved in devising effective governance systems to steer human uses of virtual reality. If anything, these difficulties are becoming more severe as the cascade of technological innovations continues. On the other hand, digital technologies may provide the means to address a variety of needs for governance, either by alleviating the underlying prob- 
lems or by devising innovative methods to move the provisions of governance systems from paper to practice. Consider the following examples to provide a preliminary sense of what is feasible in this regard.

By allowing people to work from home, take virtual classes, shop online, and engage in social interactions via Zoom or similar platforms, the use of digital technologies can reduce problems arising from congestion or crowding, thereby cutting down on needs to regulate congestion in public places and reducing emissions of pollutants including greenhouse gases. Similarly, by optimizing uses of heating and cooling systems and controlling uses of water, digital technologies can reduce the demand for energy and alleviate water shortages. Clearly, these developments will lead to major shifts in residential patterns and in urban infrastructure. Already, for example, retail establishments are being turned into warehouses for fulfilling online orders and commercial office spaces are being adjusted in response to the rise in numbers of telecommuters. The reduction in face-to-face interactions will prove harmful to some activities (e.g., live theater and concerts), and there is no doubt that virtual interactions are a poor substitute for face-to-face interactions with regard to some features of human relationships. For example, it is hard to replicate virtually the chemistry of face-to-face interactions that is so central to sparking creativity in the arts and sciences. This means that adapting to the changes brought about by the onset of the cyber age will require not only far-reaching adjustments on an ongoing basis but also sensitivity in providing continuing opportunities for face-to-face interactions. Still, this does not alter the fact that the growing use of virtual reality can play a critical role in addressing some needs for governance.

Similar observations are in order regarding the uses of digital technologies in moving governance systems from paper to practice. While authoritarian leaders desiring to control the behavior of their subjects may misuse these technologies, digital systems employing advanced observation capabilities and making use of big data can help to determine allowable harvest levels for renewable resources, operate emissions trading schemes efficiently, and assess rates of progress toward fulfilling collectively determined goals. Similarly, the use of artificial intelligence to pilot aircraft or robots to clean up after accidents can make it possible to engage in activities that would be too dangerous for real people to undertake. These applications do not constitute a panacea. Quite apart from problems arising from intentional misuses, there is plenty of scope for things to go wrong in the use of digital technologies to deal with matters involving the administration of governance systems. Nevertheless, the technologies of the cyber age open up opportunities to make use of steering mechanisms that were unavailable in earlier times. 
There are multiple links between technological innovation and the creation and administration of governance systems. There is no need to engage in technological determinism to recognize that technological innovation is a major driver of the evolution of human societies. Whether or not the pace and trajectory of technological innovations are themselves subject to effective steering is an open question. Perhaps the most promising initiatives in this realm center on efforts to exercise control over applications of new technologies in contrast to steering the course of technological innovation itself. As the massive Chinese effort to construct the Great Firewall suggests, such efforts may reflect the pursuit of objectives that are antithetical to liberal values. But this case also indicates that achieving effective control in this realm, especially in the cyber age, is easier said than done. More generally, there are serious limitations to efforts to steer when it comes to regulating the influence of a pervasive technological culture in contrast to guiding the use of technological innovations to pursue specific goals.

With regard to the role of technology in efforts to meet various needs for governance, much has been said about the debate between those who believe that technological solutions will be forthcoming when the need arises and those who fear that a faith in technological fixes is part of the problem. There is no simple way to resolve this debate. Technological innovation can play an important role in solving some problems; the dangers of an excessive faith in technological fixes are real. Perhaps more relevant is an analysis of the roles that technological innovations can play in providing early warning of emerging problems, designing regimes that are well matched to the relevant problems, administering the provisions of steering mechanisms effectively and efficiently, and helping to evaluate the performance of governance systems. Here, too, it is important to exercise caution to avoid the temptation to rely too heavily on the contributions of technology. But it seems clear that technological innovations, especially those involving the digital technologies of the cyber age, can help substantially in addressing the challenges arising in the governance of complex systems. 